\title{
Continuation methods in certain metric and geodesic spaces
}

\author{
W. A. Kirk ${ }^{1} \cdot$ Naseer Shahzad $^{2}$ (D)
}

Received: 27 February 2016 / Accepted: 14 March 2016 / Published online: 28 March 2016 (C) The Author(s) 2016. This article is published with open access at Springerlink.com

\begin{abstract}
In this paper it is shown that a classical continuation principle due to Granas for contractions holds under weaker contractive assumptions. This leads to a LeraySchauder principle for such contractions in hyperbolic spaces. Some applications to nonexpansive mappings in hyperbolic geodesic spaces are also discussed.
\end{abstract}

Keywords Contraction mappings · Nonexpansive mappings · Geodesic spaces · Hyperbolic spaces - Continuation principles - Fixed point theorem - The LeraySchauder boundary condition

Mathematics Subject Classification Primary 54H25 • 47H09

\section{Introduction}

Continuation methods in metric fixed point theory have been largely motivated by the classical Leray-Schauder condition, which was originally formulated over eighty years ago in [22] for compact mappings. Since then this condition has subsequently been extensively used to study existence of fixed points for various types of mappings.

Communicated by Ari Laptev.

$凶 \quad$ Naseer Shahzad

nshahzad@kau.edu.sa

W. A. Kirk

william-kirk@uiowa.edu

1 Department of Mathematics, The University of Iowa, Iowa City, IA 52242-1419, USA

2 Department of Mathematics, King Abdulaziz University, P. O. Box 80203, Jeddah, Saudi Arabia 
The original idea may be formulated as follows. Suppose $X$ is a Banach space with $D \subset X$. A mapping $T: D \rightarrow X$ is said to satisfy the Leray-Schauder condition if there exists $z \in \operatorname{int}(D)$ such that

$$
T(x)-z \neq \lambda(x-z) \text { for all } x \in \partial D \text { and } \lambda>1 \text {. }
$$

To place this condition in a more historical context we refer, for example, to a recent paper by Morales [23] and the references cited therein. We also refer to the recent paper by Garcia-Falset et al. [6] for additional historical comments.

In [23] Morales answered a long-standing open question posed by the first author in 1975 [15]. Specifically, it was proved in [15] that if $K$ is a bounded closed convex subset of a Banach space which has the fixed point property for nonexpansive mappings, and if $T: K \rightarrow X$ satisfies the Leray-Schauder condition relative to some point $z \in \operatorname{int}(K)$, then $T$ has a fixed point under the additional assumption that $\inf \{\|x-T(x)\|: x \in \partial K\}>0$. Morales proved, among other things, that even for a wider class of mappings this second assumption may be dropped.

The purpose of the present paper is to study the Leray-Schauder condition and related continuation methods in the context of certain metric and geodesic spaces. In particular it is shown that a classical continuation principle due to Granas for contractions holds under slightly weaker assumptions. It is also shown that if $G$ is a bounded open set in a complete hyperbolic space $X$, and if $f: \bar{G} \rightarrow X$ is nonexpansive, then $\inf \{d(x, f(x)): x \in \bar{G}\}=0$ if there exists $p \in G$ such that $x \notin(p, f(x))$ for all $x \in \partial G$. This condition, in the present context, is equivalent to the Leray-Schauder boundary condition.

We begin with the terminology of Papadopoulos [24]. Let $(X, d)$ be a metric space. Recall that a geodesic path joining $x \in X$ to $y \in X$ (or, more briefly, a geodesic from $x$ to $y$ ) is a map $c$ from a closed interval $[0, l] \subset \mathbb{R}$ to $X$ such that $c(0)=x, c(l)=y$, and $d\left(c(t), c\left(t^{\prime}\right)\right)=\left|t-t^{\prime}\right|$ for all $t, t^{\prime} \in[0, l]$. In particular, $c$ is an isometry and $d(x, y)=l$. The image $\alpha$ of $c$ is called a geodesic (or metric) segment joining $x$ and $y$. In this case for $t \in[0,1]$ we use $(1-t) x \oplus t y$ to denote the point of $\alpha$ which has distance $t d(x, y)$ from $x$. The space $(X, d)$ is said to be a geodesic space if every two points of $X$ are joined by a geodesic, and $X$ is said to be uniquely geodesic if there is exactly one geodesic joining $x$ and $y$ for each $x, y \in X$.

Many results in this paper hold in a special class of spaces called hyperbolic spaces. We turn now to the terminology of Kohlenbach [21].

Definition 1.1 $(X, d, W)$ is called a hyperbolic space if $(X, d)$ is a metric space and $W: X \times X \times[0,1] \rightarrow X$ is a function satisfying

(i) $d(z, W(x, y, \lambda)) \leq(1-\lambda) d(z, x)+\lambda d(z, y)$ for each $x, y, z \in X$ and $\lambda \in$ $[0,1]$

(ii) $d\left(W\left(x, y, \lambda_{1}\right), W\left(x, y, \lambda_{2}\right)\right)=\left|\lambda_{1}-\lambda_{2}\right| d(x, y)$ for each $x, y \in X$ and $\lambda_{1}, \lambda_{2} \in[0,1]$

(iii) $W(x, y, \lambda)=W(y, x,(1-\lambda))$ for each $x, y \in X$ and $\lambda \in[0,1]$;

(iv) $\left\{\begin{array}{l}d(W(x, z, \lambda), W(y, w, \lambda)) \leq(1-\lambda) d(x, y)+\lambda d(z, w) \\ \text { for each } x, y, z, w \in X \text { and } \lambda \in[0,1] .\end{array}\right.$ 
If only condition (i) is satisfied, then $(X, d, W)$ is a convex metric space in the sense of Takahashi [26]. Conditions (i)-(iii) together are equivalent to $(X, d, W)$ being a space of hyperbolic type in the sense of Goebel-Kirk [9]. Condition (iii) ensures that the set

$$
\{W(x, y, \lambda): \lambda \in[0,1]\}
$$

is a geodesic in the usual sense. In this case we use $(1-\lambda) x \oplus \lambda y$ to denote $W(x, y, \lambda)$. The geodesic segment joining $x$ and $y$ is denoted by $[x, y]$ (with the usual convention for $(x, y]$ and $[x, y))$. Thus $(1-\lambda) x \oplus \lambda y$ denotes the point of $[x, y]$ with distance $\lambda d(x, y)$ from $x$. A subset $Y \subseteq X$ is said to be convex if $[x, y] \subset Y$ for every $x, y \in Y$.

The relevant observation at this point is that it is not essential that geodesic segments joining each two points of $X$ be unique. It suffices to assume only that some family of geodesic segments satisfy the relevant axioms; in this instance (i)-(iii). Thus the class of spaces of hyperbolic type includes all normed linear spaces (not merely those with strictly convex norm) as well as all convex subsets thereof.

For a more detailed discussion of these concepts we refer, e.g., to Chapters 6 and 9 of Kirk-Shahzad [20].

\section{Continuation methods for contractions}

In this section we take two facts as our points of departure. The first is a following fundamental continuation principle due to Andrzej Granas. The second is an extension of Banach's contraction mapping theorem due to Felix Browder. Here, and throughout the remainder of the section, we adopt the terminology of Jachymski and Jóźwik [14].

Theorem 2.1 ([10]) Let $U$ be a domain in a complete metric space $X$, let $f, g: \bar{U} \rightarrow$ $X$ be two contraction mappings, and suppose there exists $H: \bar{U} \times[0,1] \rightarrow X$ such that

(a) $H(\cdot, 1)=f, H(\cdot, 0)=g$;

(b) $H(x, t) \neq x$ for every $x \in \partial U$ and $t \in[0,1]$;

(c) there exists $\alpha<1$ such that $d(H(x, t), H(y, t)) \leq \alpha d(x, y)$ for every $x, y \in \bar{U}$ and $t \in[0,1]$;

(d) there exists a constant $M \geq 0$ such that for every $x \in \bar{U}$ and $t, s \in[0,1]$,

$$
d(H(x, t), H(x, s)) \leq M|s-t| .
$$

Then $f$ has a fixed point if and only if $g$ has a fixed point.

Theorem 2.2 ([4]) Let $(X, d)$ be a complete metric space and suppose $f: X \rightarrow X$ satisfies

$$
d(f(x), f(y)) \leq \psi(d(x, y)) \text { for all } x, y \in X,
$$

where $\psi:[0, \infty) \rightarrow[0, \infty)$ is monotone nondecreasing, continuous from the right, and such that $\psi(t)<t$ for all $t>0$. Then $f$ has a unique fixed point $x^{*} \in X$ and moreover $f^{n}(x) \rightarrow x^{*}$ as $n \rightarrow \infty$ for every $x \in X$. 
In what follows we refer to mappings that satisfy the above condition Browder contractions with contractive function $\psi$. In his survey [13], Jacek Jachymski shows that Browder's contractive condition is equivalent to or, in fact subsumes, many contractive conditions that have subsequently appeared in the literature. By making some modifications in Marlène Frigon's proof of Theorem 2.1 (see [5]) we obtain the following extension of Granas's theorem. We point out that in her original paper, Frigon mentions that condition $(\mathrm{d})$ of Theorem 2.1 may be weakened to condition $\left(\mathrm{d}^{\prime}\right)$ below.

Theorem 2.3 Let $U$ be a domain in a complete metric space $X$, let $\psi:[0, \infty) \rightarrow$ $[0, \infty)$ be monotone nondecreasing, continuous from the right, and such that $\psi(t)<t$ for all $t>0$ and let let $f, g: \bar{U} \rightarrow X$ be two Browder contractions with common contractive function $\psi$. Suppose also that there exists $H: \bar{U} \times[0,1] \rightarrow X$ such that

(a) $H(\cdot, 1)=f, H(\cdot, 0)=g$;

(b) $H(x, t) \neq x$ for every $x \in \partial U$ and $t \in[0,1]$;

$\left(\mathrm{c}^{\prime}\right) d(H(x, t), H(y, t)) \leq \psi(d(x, y))$ for every $x, y \in \bar{U}$ and $t \in[0,1]$;

$\left(\mathrm{d}^{\prime}\right)$ there exists a continuous function $\phi:[0,1] \rightarrow \mathbb{R}$ such that for every $x \in \bar{U}$ and $t, s \in[0,1]$,

$$
d(H(x, t), H(x, s)) \leq|\phi(t)-\phi(s)| .
$$

Then $f$ has a fixed point if and only if $g$ has a fixed point.

Proof of Theorem 2.3 Let

$$
Q=\{\lambda \in[0,1]: H(\cdot, \lambda) \text { has a fixed point }\}
$$

If $g$ has a fixed point then $0 \in Q$. We now show that $Q$ is open in $[0,1]$. Let $\lambda_{0} \in Q$ and suppose $H\left(x, \lambda_{0}\right)=x$. Since $x \notin \partial U$ there exists $r>0$ such that the closed ball $B(x ; r) \subset U$. Take $\delta>0$ such that for $\left|\lambda-\lambda_{0}\right|<\delta,\left|\phi(\lambda)-\phi\left(\lambda_{0}\right)\right|<r-\psi(r)$ (where $\psi$ and $\phi$ are as in $\left(\mathrm{c}^{\prime}\right)$ and $\left.\left(\mathrm{d}^{\prime}\right)\right)$. Then the function $H(\cdot, \lambda): B(x ; r) \rightarrow$ $B(x ; r)$. Indeed, for every $y \in B(x ; r)$ we have (using $\left(\mathrm{c}^{\prime}\right)$ and $\left.(\mathrm{d})^{\prime}\right)$

$$
\begin{aligned}
d(x, H(y, \lambda)) & \leq d\left(H\left(x, \lambda_{0}\right), H\left(y, \lambda_{0}\right)\right)+d\left(H\left(y, \lambda_{0}\right), H(y, \lambda)\right) \\
& \leq \psi(d(x, y))+\left|\phi(\lambda)-\phi\left(\lambda_{0}\right)\right| \\
& \leq \psi(r)+r-\psi(r)=r .
\end{aligned}
$$

Since the restriction of $H(\cdot, \lambda)$ to the ball $B(x ; r)$ satisfies the conditions of Browder's theorem, $H(\cdot, \lambda)$ has a fixed point for each $\lambda \in[0,1]$ for which $\left|\lambda-\lambda_{0}\right|<\delta$.

To see that $Q$ is closed in $[0,1]$, suppose $\lambda_{n} \in Q, n=1,2, \ldots$, and suppose $\lambda_{n} \rightarrow \lambda$ as $n \rightarrow \infty$. For each $n \in \mathbb{N}$ there exists $x_{n} \in \bar{U}$ such that

$$
H\left(x_{n}, \lambda_{n}\right)=x_{n} .
$$

Then if $m, n \in \mathbb{N}$,

$$
\begin{aligned}
d\left(x_{m}, x_{n}\right) & \leq d\left(H\left(x_{m}, \lambda_{m}\right), H\left(x_{m}, \lambda_{n}\right)\right)+d\left(H\left(x_{m}, \lambda_{n}\right), H\left(x_{n}, \lambda_{n}\right)\right) \\
& \leq\left|\phi\left(\lambda_{m}\right)-\phi\left(\lambda_{n}\right)\right|+\psi\left(d\left(x_{m}, x_{n}\right)\right) .
\end{aligned}
$$


If $\left\{x_{n}\right\}$ is not a Cauchy sequence then by passing to a subsequence we may suppose $d\left(x_{m}, x_{n}\right) \rightarrow t>0$ as $m, n \rightarrow \infty$. Moreover, by passing to a subsequence again we may suppose that either $d\left(x_{m}, x_{n}\right) \searrow t$ or $d\left(x_{m}, x_{n}\right) \nearrow t$. In the first case, since $\psi$ is continuous from the right, $\psi\left(d\left(x_{m}, x_{n}\right)\right) \rightarrow \psi(t)$ as $m, n \rightarrow \infty$. In the second case, because $\psi$ is nondecreasing, $\psi\left(d\left(x_{m}, x_{n}\right)\right) \rightarrow r \leq \psi(t)$ as $m, n \rightarrow \infty$. In either case, this leads to the contradiction

$$
t \leq \psi(t)
$$

It follows that $\left\{x_{n}\right\}$ is a Cauchy sequence in $\bar{U}$, so there exists $x \in \bar{U}$ such that $x_{n} \rightarrow x$ as $n \rightarrow \infty$. Moreover, since $\psi(0)=0$ and $\psi$ is continuous from the right,

$$
\begin{aligned}
d\left(H(x, \lambda), x_{n}\right) & =d\left(H(x, \lambda), H\left(x_{n}, \lambda_{n}\right)\right) \\
& \leq d\left(H(x, \lambda), H\left(x, \lambda_{n}\right)\right)+d\left(H\left(x, \lambda_{n}\right), H\left(x_{n}, \lambda_{n}\right)\right) \\
& \leq\left|\phi(\lambda)-\phi\left(\lambda_{n}\right)\right|+\psi\left(d\left(x, x_{n}\right)\right) \rightarrow 0 \text { as } n \rightarrow \infty .
\end{aligned}
$$

This proves that $x_{n} \rightarrow H(x, \lambda)$ as $n \rightarrow \infty$. Therefore $H(x, \lambda)=x$, proving $\lambda \in Q$. It follows that $Q=[0,1]$, completing the proof.

Remark 1 A routine argument shows that it is possible to replace condition $\left(\mathrm{d}^{\prime}\right)$ in Theorem 2.3 with the condition

$\left(\mathrm{d}^{\prime \prime}\right)$ there exists a function $\phi:[0,1] \rightarrow \mathbb{R}$ which is continuous at 0 such that for every $x \in \bar{U}$ and $t, s \in[0,1]$,

$$
d(H(x, t), H(x, s)) \leq \phi(|t-s|)
$$

Remark 2 Theorem 2.3 extends Corollary 3.2 of Agarwal, et al. [1] in that $\psi$ is merely assumed to be continuous from the right rather than continuous.

Other weakenings in Theorem 2.1 are possible. For example, it is possible to replace Browder's condition involving the function $\psi$ in Theorem 2.2 with a condition introduced in [14] which is a minor variant of a condition due to Geraghty [7]. Suppose $\alpha: \mathbb{R}^{+} \rightarrow[0,1)$ satisfies the condition: If $\left\{t_{n}\right\}$ is bounded, then $\alpha\left(t_{n}\right) \rightarrow 1 \Rightarrow t_{n} \rightarrow 0$ as $n \rightarrow \infty$. Condition 2.1 now becomes

$$
d(f(x), f(y)) \leq \alpha(d(x, y)) d(x, y) \text { for all } x, y \in X .
$$

Mappings satisfying this condition are called Geraghty (III) contractions in [14], where it is shown that such contractions are equivalent to Browder contractions. Therefore Theorem 2.3 holds for this class of mappings upon making the following adjustment in Condition $\left(\mathrm{c}^{\prime}\right)$ :

$\left(\mathrm{c}^{\prime \prime}\right) d(H(x, t), H(y, t)) \leq \alpha(d(x, y)) d(x, y)$ for every $x, y \in \bar{U}$ and $t \in[0,1]$. We include some details of the proof to illustrate the difference in methodology.

Theorem 2.4 Let $U$ be a domain in a complete metric space $(X, d)$, let $\alpha: \mathbb{R}^{+} \rightarrow$ $[0,1)$ satisfy for bounded $\left\{t_{n}\right\}, \alpha\left(t_{n}\right) \rightarrow 1 \Rightarrow t_{n} \rightarrow 0$ as $n \rightarrow \infty$, and let $f, g: \bar{U} \rightarrow$ $X$ be two Geraghty (III) contractions with common contractive function $\alpha$. Suppose 
also that $H: \bar{U} \times[0,1] \rightarrow X$ satisfies $(a),(b),\left(c^{\prime}\right),\left(d^{\prime}\right)$ of Theorem 2.3 where $\psi(d(x, y))$ is replaced with $\alpha(d(x, y)) d(x, y)$ in $\left(c^{\prime}\right)$. Then $f$ has a fixed point if and only if $g$ has a fixed point.

Proof The main difficulty in this proof involves showing that for $\left|\lambda-\lambda_{0}\right|$ sufficiently small, $H(\cdot, \lambda): B(x ; r) \rightarrow B(x ; r)$. We prove this step in detail, adopting the notation in the proof above. To see that $Q$ is open in $[0,1]$, let $\lambda_{0} \in Q$ and suppose $H\left(x, \lambda_{0}\right)=$ $x$. Since $x \notin \partial U$ there exists $r>0$ such that the closed ball $B(x ; r) \subset U$. Take $\delta_{r}>0$ such that for $\left|\lambda-\lambda_{0}\right|<\delta_{r},\left|\phi(\lambda)-\phi\left(\lambda_{0}\right)\right|<r-\alpha(r) r$ (where $\alpha$ and $\phi$ are as in $\left(\mathrm{c}^{\prime \prime}\right)$ and $\left.\left(\mathrm{d}^{\prime}\right)\right)$. Then if $d(x, y)=r, d(x, H(y, \lambda)) \leq r$. Indeed, we have (using $\left(\mathrm{c}^{\prime \prime}\right)$ and $\left.\left(\mathrm{d}^{\prime}\right)\right)$

$$
\begin{aligned}
d(x, H(y, \lambda)) & \leq d\left(H\left(x, \lambda_{0}\right), H\left(y, \lambda_{0}\right)\right)+d\left(H\left(y, \lambda_{0}\right), H(y, \lambda)\right) \\
& \leq \alpha(d(x, y)) d(x, y)+\left|\phi(\lambda)-\phi\left(\lambda_{0}\right)\right| \\
& \leq \alpha(r) r+r-\alpha(r) r=r .
\end{aligned}
$$

Now take $0<r^{*}<r$ so that $r^{*} \leq \alpha(r) r$. Then if $d(x, y) \leq r^{*}$ and $\left|\lambda-\lambda_{0}\right|<\delta_{r^{*}}$ (and using the fact that $\alpha(d(x, y))<1)$,

$$
\begin{aligned}
d(x, H(y, \lambda)) & \leq \alpha(d(x, y)) d(x, y)+r-\alpha(r) r \\
& <d(x, y)+r-\alpha(r) r \\
& \leq r^{*}+r-\alpha(r) r \leq r .
\end{aligned}
$$

For each $r^{\prime}$ such that $r^{*}<r^{\prime}<r$ there exists $\delta_{r^{\prime}}>0$ such that if $\left|\lambda-\lambda_{0}\right|<\delta_{r^{\prime}}$, then $\left|\phi(\lambda)-\phi\left(\lambda_{0}\right)\right|<r^{\prime}-\alpha\left(r^{\prime}\right) r^{\prime}$. Hence if $d(x, y)=r^{\prime}$,

$$
d(x, H(y, \lambda)) \leq r^{\prime} \leq r .
$$

Now let $\delta=\inf \left\{\delta_{r^{\prime}}: r^{*} \leq r^{\prime} \leq r\right\}$. We now show that $\delta>0$. If $\delta=0$, then there exists a sequence $\left\{r_{n}^{\prime}\right\} \subset\left[r^{*}, r\right]$ such that $r_{n}^{\prime}-\alpha\left(r_{n}^{\prime}\right) r_{n}^{\prime} \rightarrow 0$ as $n \rightarrow \infty$. If $\alpha\left(r_{n}^{\prime}\right) \nrightarrow 1$ we may pass to a subsequence $\left\{r_{n_{k}}^{\prime}\right\}$ of $\left\{r_{n}^{\prime}\right\}$ such that $\alpha\left(r_{n_{k}}^{\prime}\right) \rightarrow t>0$ as $k \rightarrow \infty$. Then

$$
\liminf \left[r_{n_{k}}^{\prime}\left(1-\alpha\left(r_{n_{k}}^{\prime}\right)\right)\right] \geq r^{*}(1-t)>0
$$

contradicting $r_{n_{k}}^{\prime}-\alpha\left(r_{n_{k}}^{\prime}\right) r_{n_{k}}^{\prime} \rightarrow 0$ as $k \rightarrow \infty$. Therefore $\alpha\left(r_{n}^{\prime}\right) \rightarrow 1$. However $\alpha\left(r_{n}^{\prime}\right) \rightarrow 1$ implies $r_{n}^{\prime} \rightarrow 0$, and this contradicts the fact that $r_{n}^{\prime} \geq r^{*}$. Therefore it must be the case that $\delta>0$, and for each $y \in B(x ; r)$ we have $\left|\lambda-\lambda_{0}\right|<\delta \Rightarrow$ $d(x, H(y, \lambda)) \leq r$. Hence for such $\lambda, H(\cdot, \lambda): B(x ; r) \rightarrow B(x ; r)$.

The remainder of the proof is straightforward, following the method of Theorem 2.3.

Remark 3 In a subsequent paper [8], Geraghty weakened his contractive condition, requiring only that $\alpha\left(t_{n}\right) \rightarrow 1 \Rightarrow t_{n} \rightarrow 0$ for monotone decreasing sequences $\left\{t_{n}\right\} \subset \mathbb{R}^{+}$. Mappings satisfying this condition are called Geraghty (II) contractions 
in [14]. It is shown in [11] that the class of Geraghty (II) contractions coincides with a class of contractions introduced by Boyd and Wong in [2]. The Boyd-Wong contractions are similar to the Browder contractions except that no monotonicity condition is required on the Browder contractive function $\psi$, and it is only assumed that $\psi$ is upper semicontinuous from the right. It is noted in [14] that Boyd-Wong contractions properly contain the Browder contractions. For a comprehensive comparison of these and numerous related contractive conditions, we refer to the survey [14] by Jachymski and Jóźwik.

Question. We leave open the question of whether Theorem 2.3 holds for the Geraghty (II) (= Boyd-Wong) contractions.

\section{The Leray-Schauder condition for contractions in hyperbolic spaces}

The following is an application of Theorem 2.3.

Theorem 3.1 Let $G$ be a bounded domain in a complete hyperbolic space $(X, d)$ and suppose $f: \bar{G} \rightarrow X$ is either a Browder or a Geraghty (III) contraction. Suppose also that there exists $p \in G$ such that $x \notin(p, f(x))$ for all $x \in \partial G$. Then $f$ has $a$ unique fixed point in $\bar{G}$.

Proof We assume that $f$ is a Browder contraction. Thus $f: \bar{G} \rightarrow X$ satisfies

$$
d(f(x), f(y)) \leq \psi(d(x, y)) \quad \text { for all } x, y \in \bar{G}
$$

where $\psi$ is Browder contractive function. Define $H: \bar{G} \times[0,1] \rightarrow X$ as follows. For $x \in G$ and $t \in[0,1]$, take $H(x, t)=f_{t}(x)$, where $f_{t}(x)$ is the point of the segment $[p, f(x)]$ with distance $t d(p, f(x))$ from $p$. Then $H(\cdot, 1)=f$ and $H(\cdot, 0) \equiv p$. If $f$ has a fixed point in $\partial G$ there is nothing to prove, so we may assume that $x \notin(p, f(x))$ for all $x \in \partial G$. This assures that condition (b) of Theorem 2.3 holds. Also, using condition (iv) of the definition of a hyperbolic space (taking $x=y$ ) we have:

$$
\begin{aligned}
d(H(x, t), H(y, t)) & =d\left(f_{t}(x), f_{t}(y)\right) \\
& \leq t d(f(x), f(y)) \\
& \leq t \psi(d(x, y))
\end{aligned}
$$

so condition ( $\left.\mathrm{c}^{\prime}\right)$ holds. Further, condition (3.1) implies $d(f(x), f(y)) \leq d(x, y)$ for each $x, y \in G$, and because $G$ is bounded it follows that $f(G)$ is bounded. Therefore there exists $M>0$ such that $d(p, f(x)) \leq M$ for all $x \in G$. Hence by condition (ii),

$$
\begin{aligned}
d(H(x, t), H(x, s)) & =d((1-t) p \oplus t f(x),(1-s) p \oplus s f(x)) \\
& =|s-t| d(p, f(x)) \\
& \leq M|s-t|
\end{aligned}
$$


for some $M>0$, so condition ( $\left.\mathrm{d}^{\prime}\right)$ holds upon taking $\phi(s)=M s$. The conclusion of the theorem now follows from Theorem 2.3 (or Theorem 2.4 in the case that $f$ is a Geraghty (III) contraction).

Remark 4 Theorem 3.1 extends Theorem 3.3 of [1] from Banach spaces to hyperbolic spaces.

\section{Nonexpansive mappings}

Theorem 4.1 Let $G$ be a bounded domain in a complete hyperbolic space $(X, d)$ and suppose $f: \bar{G} \rightarrow X$ is a nonexpansive mapping. Suppose also that there exists $p \in G$ such that $f$ satisfies the Leray-Schauder condition: $x \notin(p, f(x))$ for all $x \in \partial G$. Then inf $\{d(x, f(x)): x \in \bar{G}\}=0$.

Proof As above, for $x \in G$ and $t \in[0,1)$, let $f_{t}(x)$ be the point of the segment $[p, f(x)]$ with distance $t d(p, f(x))$ from $p$. Then $f_{t}: \bar{G} \rightarrow X$ is a contraction mapping and $x \notin\left(p, f_{t}(x)\right)$ for each $x \in \partial G$, so by Theorem 3.1 for each such $t$ there exists $x_{t} \in \bar{G}$ such that $f_{t}\left(x_{t}\right)=x_{t}$. Thus (since $\left\{f\left(x_{t}\right)\right\}$ is bounded)

$$
\begin{aligned}
d\left(x_{t}, f\left(x_{t}\right)\right) & =d\left(f_{t}\left(x_{t}\right), f\left(x_{t}\right)\right) \\
& =d\left((1-t) p \oplus t f\left(x_{t}\right), f\left(x_{t}\right)\right) \\
& =(1-t) d\left(p, f\left(x_{t}\right)\right) \\
& \rightarrow 0 \text { as } t \rightarrow 1^{-} .
\end{aligned}
$$

Our next observation involves the CAT(0) spaces of Gromov (see [3]). It is known ([17], Theorem 21) that if $K$ is a bounded closed convex subset of a complete CAT(0) space $X$ and if $T: K \rightarrow X$ is a nonexpansive mapping for which $\inf \{d(x, f(x)): x \in K\}=0$, then $T$ has a fixed point. In conjunction with Theorem 4.1 this shows that the Leray-Schauder boundary condition implies the existence of a fixed point for such mappings if $i n t(K) \neq \emptyset$. However in this case it is known that the convexity assumption on $K$ is not even needed. The following is Theorem 3.3 of [18].

Theorem 4.2 Let $G$ be a bounded open set in a complete $C A T(0)$ space $(X, d)$, and suppose $f: G \rightarrow X$ is nonexpansive. Suppose also that there exists $p \in G$ such that $x \notin(p, f(x))$ for all $x \in \partial G$. Then $f$ has a fixed point in $\bar{G}$.

We now turn to another application. It is easy to see that if $B:=B(p ; r)$ is a closed ball in a complete hyperbolic space and if $f: B \rightarrow B(p ; r+\varepsilon)$ for some $\varepsilon>0$, then

$$
\inf \{d(x, f(x)): x \in B\} \leq \varepsilon \text {. }
$$

This is because Theorem 4.1 implies inf $\{d(x, f(x)): x \in B\}=0$ if $f$ satisfies the Leray-Schauder condition on $\partial B$ for some $x$ or, if the Leray-Schauder condition fails 
there exists a point $x \in \partial B$ such that $x \in(p, f(x))$, which implies $d(x, f(x)) \leq \varepsilon$. We now examine the extent to which this observation extends to arbitrary convex sets.

Let $K$ be a bounded closed convex subset of a complete linear hyperbolic space $X$ in the sense that each two distinct points $x, y$ of $X$ lie on a unique geodesic (metric) line containing the geodesic segment $[x, y]$. (This is the approach taken in [25]). The following is an immediate extension of the observation about approximate fixed points in closed balls. The $\varepsilon$-neighborhood of $K$ for $\varepsilon>0$ is the set:

$$
N_{\varepsilon}(K)=\{x \in X: \operatorname{dist}(x, K) \leq \varepsilon\} .
$$

Theorem 4.3 Let $K$ be a bounded closed convex subset of a complete linear hyperbolic space, and suppose int $(K) \neq \emptyset$.If $\varepsilon>0$ and $f: K \rightarrow N_{\varepsilon}(K)$ is nonexpansive, then

$$
\inf \{d(x, f(x)): x \in K\} \leq \operatorname{diam}(K)-2 \bar{r}+\varepsilon,
$$

where $\bar{r}=\sup \{r>0: B(p ; r) \subset K$ for some $p \in \operatorname{int}(K)\}$.

Proof Since int $(K) \neq \emptyset$ there exist $p \in \operatorname{int}(K)$ and $r>0$ such that $B(p ; r) \subset K$ and $B\left(p ; r^{\prime}\right) \nsubseteq K$ if $r^{\prime}>r$. Let $\varepsilon^{\prime}>\varepsilon$ be arbitrary. If the Leray-Schauder condition holds on $\partial K$ relative to $p$ then by Theorem 4.1 there is nothing to prove. Otherwise there exists $y \in \partial K$ such that $y \in(p, f(y))$. Also, since $f(y) \in N_{\varepsilon}(K)$ there exists $z \in \partial K$ such that $d(f(y), z) \leq \varepsilon^{\prime}$. If $z=y$ we are finished. Otherwise, there is a point $q$ on the geodesic line passing through $p$ and $z$ such that $d(q, p)=r$ and $d(q, p)+d(p, z)=d(q, z) \leq \operatorname{diam}(K)$. Thus $d(p, z) \leq \operatorname{diam}(K)-r$. Since $d(p, y) \geq r$, it follows that

$$
\begin{aligned}
d(y, f(y)) & =d(p, f(y))-d(p, y) \\
& \leq d(p, z)+d(z, f(y))-d(p, y) \\
& \leq d(p, z)+\varepsilon^{\prime}-d(p, y) \\
& \leq[\operatorname{diam}(K)-r]+\varepsilon^{\prime}-d(p, y) \\
& \leq \operatorname{diam}(K)-2 r+\varepsilon^{\prime} .
\end{aligned}
$$

Since $B(p ; r)$ is an arbitrary ball in $K$ and $\varepsilon^{\prime}>\varepsilon$ is arbitrary, the conclusion follows.

We do not know whether the estimate in Theorem 4.3 is optimal. Indeed, as we show in the Appendix a sharper estimate holds in a Banach space.

\section{Further remarks}

The so-called $\mathbb{R}$-trees (or metric trees) are a very special case of the CAT(0) spaces (see [3, p.167]).

Definition 5.1 An $\mathbb{R}$-tree is a metric space $X$ such that 
(i) there is a unique geodesic segment (again, denoted by $[x, y]$ ) joining each two points $x, y \in X$

(ii) if $[y, x] \cap[x, z]=\{x\}$ then $[y, x] \cup[x, z]=[y, z]$.

The following is another result of [18]. In this instance boundedness of the domain may be replaced by geodesic boundedness.

Theorem 5.1 ([18]) Let $(X, d)$ be a complete $\mathbb{R}$-tree, suppose $K$ is a closed, geodesically bounded, convex subset of $X$, and suppose $p \in \operatorname{int}(K)$. If $f: K \rightarrow X$ is continuous and satisfies the Leray-Schauder condition:

$$
x \notin(p, f(x)) \text { for all } x \in \partial K .
$$

Then $f$ has a fixed point.

Crucial to the proof of the above result is the fact that a continuous self-mapping of a geodesically bounded closed convex subset of a complete $\mathbb{R}$-tree always has a fixed point. For a detailed discussion, see [19].

Finally, we remark in passing that it is shown in [12] that for a nonexpansive mapping $T: B \rightarrow H$, where $B$ is the unit ball in a Hilbert space $H$, the existence of a fixed point for $T$ and the Leray-Schauder condition are mutually exclusive alternatives, and that this fact characterizes Hilberts space among Banach spaces. It is not clear whether a similar fact characterizes CAT(0) spaces among Busemann spaces.

Open Access This article is distributed under the terms of the Creative Commons Attribution 4.0 International License (http://creativecommons.org/licenses/by/4.0/), which permits unrestricted use, distribution, and reproduction in any medium, provided you give appropriate credit to the original author(s) and the source, provide a link to the Creative Commons license, and indicate if changes were made.

\section{Appendix}

The following is a Banach space extension of Theorem 4.3. However we do not even know whether this estimate is optimal.

Theorem 6.1 ([16]) Let $K$ be a bounded closed convex subset of a Banach space $X$ with int $(K) \neq \emptyset$, and let $f: K \rightarrow N_{\varepsilon}(K)(\varepsilon>0)$ be nonexpansive. Then

$$
\inf \{\|x-f(x)\|: x \in K\} \leq\left(\frac{\operatorname{diam}(K)-\bar{r}+\varepsilon}{\bar{r}+\varepsilon}\right) \varepsilon
$$

where

$$
\bar{r}=\sup \{r>0: B(p ; r) \subset K \text { for some } p \in i n t(K)\}
$$

Proof Because [16] may not be readily available we include the details. Since int $(K) \neq \emptyset$ there exists $r>0$ such that $B(p ; r) \subset K$, and we may further suppose $p=0$. If the Leray-Schauder condition holds for $f$ on $\partial K$ relative to $p$ then by 
Theorem 4.1 (or earlier Banach space results) there is nothing to prove, so we suppose that for some $y \in \partial K$ and $\lambda>1, f(y)=\lambda y$. Now let $\varepsilon^{\prime}>\varepsilon>0$ be arbitrary. Since $f(y) \in N_{\varepsilon}(K)$ there exists $z \in \partial K$ such that $d(f(y), z) \leq \varepsilon^{\prime}$.

Let $w \in X$ satisfy

$$
y=\left(1-\lambda^{-1}\right) w+\lambda^{-1} z
$$

Then, since $y=\lambda^{-1} f(y)$, we have

$$
\left(1-\lambda^{-1}\right) w=\lambda^{-1}(f(y)-z) .
$$

From (6.2),

$$
\begin{aligned}
\left(1-\lambda^{-1}\right)\|w\| & =\lambda^{-1}\|f(y)-z\| \\
& \leq \lambda^{-1} \varepsilon^{\prime}
\end{aligned}
$$

and it follows that

$$
\left(1-\lambda^{-1}\right)\left[\|w\|+\varepsilon^{\prime}\right] \leq \varepsilon^{\prime} .
$$

Multiplying both sides by $\|f(y)\|$ we have

$$
\left(1-\lambda^{-1}\right)\left[\|w\|+\varepsilon^{\prime}\right]\|f(y)\| \leq \varepsilon^{\prime}\|f(y)\| .
$$

Since $\left(1-\lambda^{-1}\right)\|f(y)\|=\|y-f(y)\|$,

$$
\|y-f(y)\| \leq \frac{\varepsilon^{\prime}\|f(y)\|}{\|w\|+\varepsilon^{\prime}} .
$$

However $\|f(y)\| \leq \operatorname{diam}(K)-r+\varepsilon$, so

$$
\|y-f(y)\| \leq \frac{\varepsilon^{\prime}\|f(y)\|}{\|w\|+\varepsilon^{\prime}} \leq\left(\frac{\operatorname{diam}(K)-r+\varepsilon}{\|w\|+\varepsilon^{\prime}} \varepsilon^{\prime}\right) .
$$

Also, since both $y$ and $z$ lie on $\partial K$, it follows that $w \notin \operatorname{int}(K)$. In particular $\|w\| \geq r$. Therefore

$$
\|f(y)-y\| \leq\left(\frac{\operatorname{diam}(K)-r+\varepsilon}{r+\varepsilon^{\prime}}\right) \varepsilon^{\prime} .
$$

Since $\varepsilon^{\prime}>\varepsilon$ is arbitrary and $B(p, r)$ is an arbitrary ball in $K$, the conclusion follows.

It is easy to check that $\left(\frac{\operatorname{diam}(K)-\bar{r}+\varepsilon}{\bar{r}+\varepsilon}\right) \varepsilon \leq \operatorname{diam}(K)-2 \bar{r}+\varepsilon$. This is because $\operatorname{diam}(K) \geq 2 r$ if $B(p ; r) \subset K$, with equality holding only if $\operatorname{diam}(K)=$ 
$2 r$. Thus in general the estimate in Theorem 6.1 is better that the one given by Theorem 4.3. At the same time, if $K$ is a closed ball each estimate reduces to

$$
\inf \{d(x, f(x)): x \in K\} \leq \varepsilon .
$$

\section{References}

1. Agarwal, R.P., Cho, Yoel Je, O'Regan, D.: Homotopy type results for nonlinear contractions on spaces with two metrics. In: Cho, Y.J., Kim, J.K., Kang, S.M. (eds.) Fixed Point Theory and Applications, Vol. 5, pp. 1-9. Nova Sci. Publ., Hauppauge, NY (2004)

2. Boyd, D.W., Wong, J.S.W.: On nonlinear contractions. Proc. Am. Math. Soc. 20, $458-464$ (1969)

3. Bridson, M., Haefliger, A.: Metric Spaces of Non-Positive Curvature. Springer, Berlin (1999)

4. Browder, F.: On the convergence of successive approximations for nonlinear functional equations. Nederl. Akad. Wetensch. Proc. Ser. A 71 = Indag. Math. 30, 27-35 (1968)

5. Frigon, M.: On continuation methods for contractive and nonexpansive mappings. In: Domínguez Benavides, T. (ed.) Recent Advances on Metric Fixed Point Theory, pp. 19-30. Universidad de Sevilla, Seville (1996)

6. Garcia-Falset, J., Hernándeź-Lineares, C., Mleşnite, O.: The Leray-Schauder condition in the coincidence problem for two mappings. J. Nonlinear Convex Anal. 16, 1905-1924 (2015)

7. Geraghty, M.A.: On contractive mappings. Proc. Am. Math. Soc. 40, 604-608 (1973)

8. Geraghty, M.A.: An improved criterion for fixed points of contraction mappings. J. Math. Anal. Appl. 48, 811-817 (1974)

9. Goebel, K., Kirk, W.A.: Iteration processes for nonexpansive mappings. In: Singh, S.P., Thomeier, S., Watson, B. (eds.) Topological Methods in Nonlinear Functional Analysis (Toronto, Ont., 1982), pp. 115-123. Contemp. Math., 21, Amer. Math. Soc., Providence (1983)

10. Granas, A.: Continuation methods for contractive maps. Topol. Methods Nonlinear Anal. 3, 375-379 (1994)

11. Hegedüs, M., Szilágyi, T.: Equivalent conditions and a new fixed point theorem in the theory of contractive type mappings. Math. Japon. 25(1), 147-157 (1980)

12. Ireland, M., Kirk, W.A., Sims, B.: The Leray-Schauder alternative for nonexpansive maps from the ball characterize Hilbert space. In: Takahashi, W., Tanaka, T. (eds.) Nonlinear Analysis and Convex Analysis (Niigata, 1998), pp. 167-172. World Sci. Publ., River Edge, NJ (1999)

13. Jachymski, J.: Around Browder's fixed point theorem for contractions. J. Fixed Point Theory Appl. 5, 47-61 (2009)

14. Jachymski, J., Jóźwik, I.: Nonlinear contractive conditions: a comparison and related problems. In: Jachymski, J., Reich, S. (eds.) Fixed Point Theory and its Applications, pp. 123-146. Banach Center Publ., 77, Polish Acad. Sci., Warsaw (2007)

15. Kirk, W.A.: Fixed point theorems for nonexpansive mappings satisfying certain boundary conditions. Proc. Am. Math. Soc. 50, 143-149 (1975)

16. Kirk, W.A.: Approximate fixed points for mappings in Banach spaces. In: Singh, S.P. (ed.) Nonlinear functional analysis and its applications (Maratea, 1985), pp. 299-303. NATO Adv. Sci. Inst. Ser. C Math. Phys. Sci., 173, Reidel, Dordrecht (1986)

17. Kirk, W.A.: Geodesic geometry and fixed point theory. In: Girela Álvarez, D., López Acedo, G., Villa Caro, R. (eds.) Seminar of Mathematical Analysis (Malaga/Seville, 2002/2003), pp. 195-225. Colecc. Abierta, 64, Univ. Sevilla Secr. Publ., Seville (2003)

18. Kirk, W.A.: Fixed point theorems in CAT(0) spaces and $\mathbb{R}$-trees. Fixed Point Theory Appl. 2004(4), 309-316 (2004)

19. Kirk, W.A.: Fixed points in arcwise connected spaces. In: Espínola, R., Petruşel, A., Prus, S. (eds.) Fixed Point Theory and its Applications, pp. 27-32. Casa Cărţii de Ştiinţă, Cluj-Napoca (2013)

20. Kirk, W.A., Shahzad, N.: Fixed Point Theory in Distance Spaces, p. xii+173. Springer, Cham (2014)

21. Kohlenbach, U.: Some logical metatheorems with applications in functional analysis. Trans. Am. Math. Soc. 357(1), 89-128 (2005)

22. Leray, J., Schauder, J.: Topologie et équations fonctionelles. Ann. Sci. École Norm. Sup. 51, 45-78 (1934) 
23. Morales, C.H.: The Leray-Schauder condition for continuous pseudo-contractive mappings. Proc. Am. Math. Soc. 137, 1013-1020 (2009)

24. Papadopoulos, A.: Metric spaces, convexity and nonpositive curvature. In: IRMA Lectures in Mathematics and Theoretical Physics, 6. European Mathematical Society (EMS), Zürich, pp. xii+287 (2005)

25. Reich, S., Shafrir, I.: Nonexpansive iterations in hyperbolic spaces. Nonlinear Anal. 15, 537-558 (1990)

26. Takahashi, W.: A convexity in metric space and nonexpansive mappings. I. Kodai Math. Sem. Rep. 22, 142-149 (1970) 